\title{
The Optimization Model of Flat Folding Table
}

\author{
Tian'e Wang, Jian $\mathrm{Li}^{*}$, Taiyang Niu and Baiqiang Guo \\ Information Technology College, Jilin Agricultural University, Changchun, 130118, China \\ ${ }^{*}$ Corresponding author
}

\begin{abstract}
- with the rapid development of industry design, the convenience and beautiful degree of the table got the attention of people, due to the folding table is easy to use and easy to transport, it has become an indispensable furniture in hotels, exhibitions and other places. Flat folding table design concept is single, so this article analyzes the characteristic of the existing flat folding table, we make the design convenience, stability and flexibility and also make the design way of the folding table more diverse. We establish the appropriate optimization model and establish model of MATLAB program. By solving the problems, it shows that the rationality of the model.
\end{abstract}

Keywords- flat folding table; parameter equation; optimization model; the equation of state

\section{INTRODUCTION}

As the development of design industry, for the convenience of the table and beautiful degree of our demand are higher and higher. However, at present most of the table in any ways are still not fully meet the requirements of people. In order to improve the convenience of folding table and easy to carry. It is necessary and urgent to research on a reasonable flat folding table optimization model.

In this paper, based on the rationality and demand of the flat folding table, we establish parameter equation, the equation of state and optimization model. Using MATLAB programming to solve the problems and analyze the results, we plan reasonable flat folding table scheme.

\section{PROBLEM DESCRIPTION}

In order to better meet the demand for the table in the beautiful and portable, many companies produce the tables that can be folded to ease the inconvenience. Then, table legs consist of a number of sticks, they are divided into two groups, each group with a steel bar connects woods. The both ends of steel bar respectively fixed on the outside table legs, and along the wood's has free slots to ensure the freedom of sliding, we make it aesthetically pleasing.

In the process of flat folding table mathematical model, considering the stability of the folding table, processing convenience, how much material that we need. By observing the folding process of folding table, we find that the process can be considered as steel bars turn around the outer table leg hinge that formed to do with radius of rotation of the legs for half of the circle. Folding process of folding table is regarded as continuous change, we use the Pythagorean theorem and the knowledge of trigonometry to determine the angle formula of between the desktop and legs, with round desktop as a benchmark, the spatial coordinate system is established. For the determination of the stability of the folding table, it is determined by the lateral table leg by force. Therefore the table are in a stationary state, according to the table legs on each point in different directions of torque equal, we determine the angle scope and friction of between the table legs and the desktop, we can determine the optimal angle. After setting proportion that steel bars split the table legs. Making the slot least and table leg as short as possible, the process will be more convenient. To use material minimum as objective, we make the optimal parameters and unknown variables as constraint conditions to achieve the goal of optimization. So we can determine the processing parameter of optimal design.

\section{The ESTABlishment OF THE MODELS}

According to the requirements and the analysis of the problems, in the cases of determine of table height, plate length, plate width, plate thickness, location of steel bars and the condition of the radius of the circular desktop, for determination of table all legs folded angles of flat folding and the length of each card slot, it is controlled by the angle $\alpha$ of lateral leg and the desktop and the length $w$ of the lateral wood of desktop.

When the tables are in a fixed state, the vertical distance of intersection of wood and steel bars is equal to the horizontal distance of $x$. So given the angles between lateral leg and the desktop and the length of the outside piece of desktops, we can calculate the angles between the desktops and sticks.

If the wooden desk surface is seen as a continuous linear surface, the surface of the legs is made up of numerous lines of tables' leg. Therefore, the length of the slot is the difference between starting coordinates and end coordinates in the process of folding, and then according to the coordinates of edge points of table leg to solve equation of state of the edge lines for table legs .

This paper involves the related symbols that are defined as follows:

Finally, complete content and organizational editing before formatting. Please take note of the following items when proofreading spelling and grammar:

a:half of rectangle plate long;

$b$ half of rectangle plate width;

R:radius of circular desktop;

$k$ :the table height after folding;

$x$ :distance between board edge and y axis; 
Whalf of lateral length of the wood for circular desktop;

$r$ :half of the length of the lateral table legs;

$h$ :the vertical distance between steel bars and the edge of desktop;

l:the horizontal distance from $\mathrm{x}$ axis to the steel bars;

$d:$ the horizontal distance from desktop edge to steel bars;

$s_{i}$ batten slot length of table legs;

$\alpha$ :horizontal angle between lateral table legs and desktop;

$\theta$ :horizontal angle between table legs and desktop.

We know the length of rectangular plate is $2 \mathrm{a},{ }^{\alpha}$ that is the distance between lateral leg line and desktop and $2 w$ is the length of lateral line are as known variables. We named $p$ is the ratio between the length ${ }^{r}$ of desktop edge and the length $a-w-r$ that is from steel bar to desktop.

Namely:

$$
\frac{r}{a-w-r}=p
$$

Reduction:

$$
r=\frac{p(a-w)}{1+p}
$$

The ratio between the vertical distance $h$ and the length $r$ from desktop to steal bars is the sine value from horizontal angle $\boldsymbol{\alpha}$ steel bar to outer table legs.

That is:

$$
h=r \cdot \sin \alpha=\frac{p(a-w)}{1+p} \cdot \sin \alpha
$$

The length from $x$ axis to horizontal distance of steel bars is the sum of vertical distance of the intersection point and the half length $w$ of the lateral line.

That is:

$$
l=r \cdot \cos \alpha+w=\frac{p(a-w)}{1+p} \cdot \cos \alpha+w
$$

The length $\mathrm{d}$ from board edge to the horizontal distance of steel bars is the 1 subtraction between $\mathrm{x}$ axis to horizontal distance, so we can get the distance from the edge of the desktop to $\mathrm{x}$ axis. According to the Pythagorean theorem, the square of the distance from $\mathrm{x}$ to the edge of the desktop is equal to the subtraction between square of circular radius $\mathrm{R}$ of desktop and the square of $x$ of the distance to of $y$ axis.

That is:

$$
d=l-\sqrt{R^{2}-x^{2}}=\frac{p(a-w)}{1+p} \cdot \cos \alpha+w-\sqrt{R^{2}-x^{2}}
$$

The ratio of between the vertical distance between desktop and steel bars $\mathrm{h}$ and horizontal distance $\mathrm{d}$ from board edge to horizontal distance of steel bars is tangent value of ${ }^{\theta}$ between table leg and desktop.

That is:

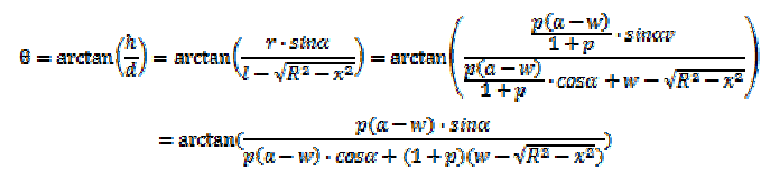

To sum up, the parameter equation is obtained as follows:

$$
\left\{\begin{array}{l}
r=\frac{p(a-w)}{1+p} \\
h=\frac{p(a-w)}{1+p} \cdot \sin \alpha \\
l=\frac{p(a-w)}{1+p} \cdot \cos \alpha+w \\
u=\frac{p(a-w)}{1+p} \cdot \cos u+w-\sqrt{R^{2}-x^{2}} \\
\theta=\arctan \left(\frac{p(a-w) \cdot \sin \alpha}{p(a-w) \cdot \cos \alpha+(1+p)\left(w-\sqrt{R^{2}-x^{2}}\right)}\right)
\end{array}\right.
$$

\section{THE SOLUTION OF EXAMPLES}

With the data of national college students' mathematical modeling competition B in 2014, the optimization model is built to find the solution to this instance. We combine with MATLAB software coding and get a reasonable design processing parameter. The concrete implementation process is shown as follows:

With a given rectangle plate size $120 \mathrm{~cm}$ by $50 \mathrm{~cm}$ by $3 \mathrm{~cm}$, each crate wide is $2.5 \mathrm{~cm}$, folding table's height is $53 \mathrm{~cm}$. We get the equation of edge of the table leg.

$$
\left\{\begin{array}{c}
x 2=x 1 \\
y 2=y 1+\cos \theta(a-y 1) \\
z 2=\sin \theta(a-y 1)
\end{array}\right.
$$


The coordinates and slot length are shown in Table 1.

TABLE I . THE COORDINATES AND SLOT LENGTH

\begin{tabular}{|c|c|c|c|c|c|c|c|c|c|c|}
\hline & 1 & 2 & 3 & 4 & 5 & 6 & 7 & 8 & 9 & 10 \\
\hline Starting point & 38.33 & 29.11 & 24.92 & 22.03 & 19.89 & 18.29 & 17.13 & 16.33 & 15.86 & 15.70 \\
\hline the end point & 38.33 & 34.71 & 33.78 & 33.43 & 33.33 & 33.35 & 33.41 & 33.47 & 33.52 & 33.53 \\
\hline \multirow[t]{2}{*}{ Slot length } & 0 & 5.60 & 8.86 & 11.40 & 13.44 & 15.05 & 16.27 & 17.14 & 17.65 & 17.82 \\
\hline & 11 & 12 & 13 & 14 & 15 & 16 & 17 & 18 & 19 & \\
\hline the end point & 33.52 & 33.47 & 33.41 & 33.35 & 33.33 & 33.43 & 33.78 & 34.71 & 38.33 & \\
\hline slot length & 17.65 & 17.14 & 16.27 & 15.05 & 13.44 & 11.40 & 8.86 & 5.60 & 0 & \\
\hline
\end{tabular}

\section{CONCLUSION}

In this paper, it based on the data of 2014 national college students' mathematical contest in modeling, using the established optimization model, we process and applicate the data. With the help of MATLAB software powerful solution, we obtain satisfactory results. In addition, this model can be applied in practical problems, we can use it to solve the problem of flat folding table design. To some extent it has practical significance.

\section{REFERENCES}

[1] Miao Zhihong Ma Jinqiang. MATLAB object-oriented program design, electronic industry press, 1989:43 to 52

[2] Xia Shengxiang,Yu Zhengwen. Commonly used numerical algorithm and MATLAB, Qing Hua university press, 1992:25 to 33

[3] Ma Changfeng. Optimization method and Matlab programming, science press, 1998:10-42 